\title{
Simultaneous 3D ESPI using a 3CCD Camera
}

\author{
Xiaodong $\mathrm{Li}^{1}$, Yimin $\mathrm{Ye}^{1}$, and Dongsheng Zhang ${ }^{1,2^{*}}$ \\ ${ }^{1}$ Shanghai Institute of Applied Mathematics and Mechanics, \\ School of Mechanics and Engineering Science, Shanghai University, Shanghai, 200444, China; \\ ${ }^{2}$ Shanghai Key Laboratory of Mechanics in Energy Engineering, \\ Shanghai, 200072, China \\ *donzhang@staff.shu.edu.cn
}

Introduction. ESPI is a non-contact, high-accuracy method for deformation measurement in many industrial fields. Due to the fact that its displacement sensitivity is closely related to the optical arrangement ${ }^{(1)}$, it is difficult to measure the $3 \mathrm{D}$ deformation simultaneously. In this paper, an apparatus which enables displacement measurement for in-plane and out-of-plane components has been developed.

Principle. The optical arrangement is illustrated in the Fig. 1. The object surface is illuminated with 3 laser beams with multiple wavelengths which include 473 $\mathrm{nm}, 532 \mathrm{~nm}$, and $671 \mathrm{~nm}$. The red and green coherent lights are divided into two beams individually (denoted as RL and GL) to illuminate the surface of the stressed object, while the blue laser (denoted as BL) passes through a Michelson Interferometer (denoted as MI) to reach the object surface. A prism-based 3CCD camera, instead of a conventional monochrome CCD camera $^{(2)}$, is adopted to acquire images before and after loading in the experimental setup. Inside a prism-based camera, since the incident light is re-directed to 3 independent sensor arrays by passing through a prism, the color coupling problem is solved and it is possible to acquire 3 images with specific wavelengths at one shot. In this paper, the real-time inspection has been achieved by combining the 4-step temporal phase-shift process ${ }^{(3)}$.

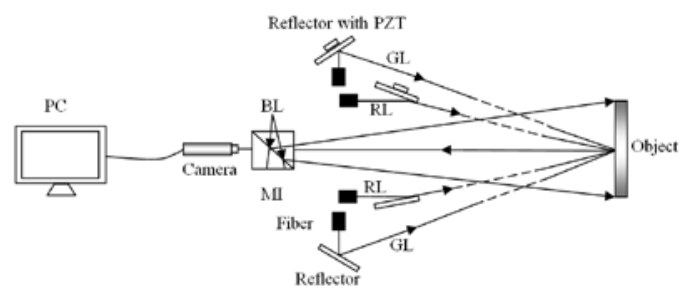

Fig. 1 Scheme of the apparatus

Verification. A rectangular constrained plate with diameter of $80 \mathrm{~mm}$ was used as the specimen. It was subjected to a normal concentrated load at the center as well as an in-plane rigid rotation. The coherent lights with wavelength of $473 \mathrm{~nm}, 532 \mathrm{~nm}$, and $671 \mathrm{~nm}$ were scattered onto the specimen surface. Images before and after loading were captured with a 4-step phase shifting. The interference phase maps of the out-of-plane and inplane are shown in Fig. 2.

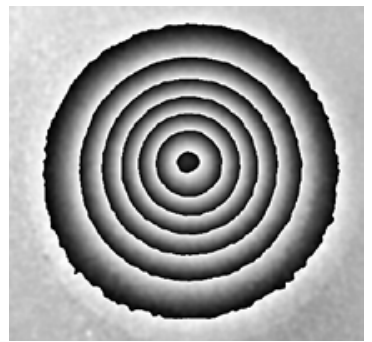

(a)

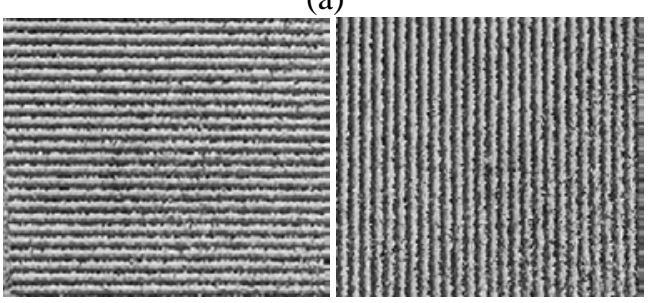

(b)

(c)

Fig. 2 Experiment results (a) out-of-plane, (b) in-plane horizontal, (c) in-plane vertical

Conclusion. In this paper, an optical arrangement which enables phase measurement for in-plane and outof-plane displacement components has been developed. Combining a prism-based 3CCD camera and multiple coherent lights with different wavelengths, the 3D displacement components have been measured simultaneously.

This work is supported by the National Key R\&D Program of China \# 2018YFF01014200, National Natural Science Foundation of China through grants \#11672347, \#11727804 and \#51732008.

\section{References.}

(1) $\mathrm{Ng} \mathrm{T} \mathrm{W.} \mathrm{ESPI} \mathrm{with} \mathrm{structured} \mathrm{illumination[J].}$ Optics and Lasers in Engineering, 2003, 40(56):553-561.

(2) Liuxing S, Yingjie Y, Wenjing Z. 3D deformation measurement based on colorful electronic speckle pattern interferometry[J]. Optik - International Journal for Light and Electron Optics, 2015, 126(23):3998-4003.

(3) Yang L, Xie X, Zhu L, et al. Review of Electronic Speckle Pattern Interferometry (ESPI) for Three Dimensional Displacement Measurement[J]. Chinese Journal of Mechanical Engineering, 2014, 27(1):1-13. 